\title{
Circuit exercise promotes health related factors in sarcopenic obesity elderly: a mini-review
}

\begin{abstract}
Sarcopenic obesity is a physiological problem caused by aging, such as reduced body function, increase of body fat, and decrease of muscle mass aggravates the quality of life of the elderly. Furthermore, it causes various social and economic problems such as degenerate and contraction of a chronic disease, increased health care costs, and increased prevalence or mortality rate. In particular, it adversely affects the condition for metabolic diseases such as lowering of body function and physical strength, blood lipids, and inflammation of the body and hormones. Currently, exercise therapy is recommended as a way to address the health problems of the elderly. In general, regular aerobic exercise is used as a way to resolve obesity and regular resistance exercise is effective in improving body function and muscle mass. Recently, the importance of resistance exercise has been emphasized in obese older people to improve muscle function and to prevent falls. However, resistance exercise has certain limitations such as risk of injury, limitation of place, and reduces obesity, so a new exercise method should be presented to the sarcopenic obese elderly. The circuit exercise using weight bearing has less risk of injuries, and it is possible to carry out the group exercise without restriction of cost and place, and it is possible to perform continuously with interest. Also, circuit exercise is recommended for the elderly because it shows improvement in body composition and in various physical factors in a relatively short time. Despite these positive effects, the studies reported so far are limited. To elucidate the effects of circuit exercise in sarcopenic obesity elderly, future studies are needed to confirm the effects of various intensity, frequency, time, and duration of exercise.
\end{abstract}

Keywords: sarcopenic obesity, circuit exercise, elderly, health related factors
Volume 3 Issue 3 - 2018

\author{
Won-Sang Jung,' Hun-Young Park ${ }^{2}$ \\ 'Department of Physical Education, Kyunghee University, Korea \\ 2Physical Activity and Performance Institute (PAPI), Konkuk \\ University, Korea
}

Correspondence: Hun-Youn Park, PhD, Physical Activity \& Performance Institute, Konkuk University, I 20, Neungdong-ro, Gwangjin-gu, Seoul 05029, Korea, Tel +821033204169, Email parkhy1980@konkuk.ac.kr

Received:May 21, 2018| Published: June 04, 2018

\section{Introduction}

Sarcopenic obesity is a compound word of sarcopenia and obesity Today, sarcopenia definition includes low muscle mass, weak muscle strength and poor physical function, although the clinical definition varies worldwide. Sarcopenic obesity is a physiological problem caused by aging, such as reduced body function, increased body fat, and reduced muscle mass aggravates the quality of life of the elderly. In particular, sarcopenic obesity causes various socio-economic problems such as chronic illness, worsening of ill health, increase of medical expenses, and mortality rate. Today, the health issue of sarcopneic obesity elderly is recognized as an important social issue.

Exercise therapy is being used as a way to solve various health problems of the elderly. Usually, regular aerobic exercise is used as a method to resolve obesity and regular resistance exercise is an effective intervention in improving body function and muscle mass. In far, many previous studies verified that regular aerobic exercise is effective in improving obesity and metabolism related variables, and resistance exercise has a powerful effect on enhancing muscle strength, muscle mass, mobility and daily physical activity. ${ }^{2}$ From the above viewpoint, the importance of resistance exercise training is emphasized as the effective modality to improve muscle function in the sarcopenic obese elderly. However, resistance exercise has several limitations such as the risk of injury, limitation of place, and reduces obesity, therefore a new exercise method for health promotion of the sarcopenic obese elderly should be suggested. ${ }^{3}$ In the case of sarcopenic obesity elderly they should be conducted circuit exercise program composed of resistance and aerobic exercise.
Therefore, this review summarizes recent research evidences suggesting that the improvement of muscle mass and body fat mass via circuit exercise program might be a valuable and viable "sarcopenic obesity therapeutic modality.

\section{Sarcopenic obesity}

Today, many researchers is conducting a variety of studies related to aging and in particular, they focus on changes in body composition such as the increase of body fat and the decrease of free fat mass and muscle strength by aging process. The most representative theme of these studies is obesity of the elderly with increase of body fat. 2 In general, along with the increase in the elderly population, the prevalence of obesity in the elderly also increases. In Korea, 1 out of 3 elderly people are obese. ${ }^{4}$ In the case of the elderly, body fat is gradually increasing even with normal aging and these changes is a risk factor for chronic diseases and increase of health problems. ${ }^{5}$ For the elderly, obesity causes a variety of serious illness that can lead to death such as depression of physical function, physical and mental stress, decrease of quality of life, sleep apnea, degenerative arthritis, hypertension, diabetes, hyperlipidemia, metabolic syndrome, and cardiovascular disease. ${ }^{6-9}$ Recently, research on the relationship between obesity and cancer have also been reported. ${ }^{10}$ Therefore, there is a great demand for care and management of obesity occurring in the elderly.

Sarcopenia, means decreased muscle mass and decreased muscle strength with increased body fat, is recognized as an important link in aging. ${ }^{11,12}$ Muscle weight is gradually reduced from the age of 
40 , accelerates after age of 60 , and about $2 \%$ of muscle mass is lost annually. ${ }^{13}$ Muscle strength is reduced more rapidly than muscle mass, decreasing by $3 \%$ per year from age of 60 , decreasing by up to $40 \%$ in ten years for older people aged over 70 who have experienced falls. ${ }^{14}$ As aging progresses, muscular strength and muscle strength are inevitably reduced, however, sarcopenia is becoming more important due to the general problems of physical function such as falls and disability of daily living activity. ${ }^{11,13}$

There are many ways to diagnose sarcopenia, but in 1998, Baumgartner's method of dividing the appendicular skeletal muscle mass (ASM) by square of the height $\left(\mathrm{m}^{2}\right)$ is known to be the most common method in Asia. ${ }^{15}$ In recent, the European Working Group on Sarcopenia in Older People (EWGSOP), an European study group for the study of sarcopenia, has included the measurement of muscular strength as well as muscle strength and physical performance (gait). ${ }^{16}$ Although standardized measurement methods and field application criteria have not been established, Baumgartner and EWGSOP methods are commonly used.

The co-occurrence of sarcopenia and obesity (vicious cycle) in the elderly leads to greater health deterioration than sarcopenia or obesity affecting independently. Recently, sarcopenic obesity has become an important research topic for solving the problems of aging society.,16

\section{Resistance exercise training}

For the elderly, resistance exercise is known to be the most effective method for an increase in muscle mass and strength.16 Previous studies have shown that resistance exercise in the elderly is not limited to physical effects but also has a positive effect on metabolic risk factors such as insulin sensitivity and glycated hemoglobin. ${ }^{17-19} \mathrm{It}$ is also known to lower inflammation levels and increase bone mineral density in the body. ${ }^{20}$ Verdijk et al., ${ }^{21}$ investigated the effect of 12 weeks of resistance exercise in the elderly men subjects aged 65 85 years. All participants performed resistance exercise three times per week and exercise intensity was $80 \%$ of one-repetition maximum (1$\mathrm{RM})$. As a result, the area of the femur muscle was increased and the muscle strength was improved. Kryger \& Andersen $^{22}$ also reported older men and women over 85 years that the size and strength of type II fibers were increased in the same period and intensity. In general, the effect of exercise on elderly people is lower than that of young adults, but regardless of gender and age, resistance exercise by elderly people is found to be positive effective for health factors. Also the reason for the obvious effect in previous studies is the high intensity of resistance exercise. ${ }^{21,22}$ According to reports, regardless of gender and age moderate-intensity resistance exercise is desirable for elderly people with weak muscles, and proper intensity is known to be more than $65 \%$ of $1 \mathrm{RM}^{23}$ As described above, when resistive exercise is performed on the elderly, not only the muscle mass and muscle strength increase but there is also the positive effect on problem of metabolism.

Resistance exercises using weight lifting devices such as barbells and dumbbells are effective in improving the muscle strength of the elderly, but sarcopenic obese elderly is associated with the risk of injury, limitations of location, and disadvantages of boredom and so other exercise methods are needed. ${ }^{24}$ The circuit exercise using weight bearing is less risk of injuries, and it is possible to carry out the group exercise without restriction of cost and place, and it is possible to perform continuously with interest. ${ }^{25}$ In addition, circuit exercise program can improve body composition and promotes various health related factors in a relatively short time and it is expected as a proficient alternative modality for the sarcopenic obesity elderl

\section{Circuit exercise training}

For the elderly, three types of exercise are recommended: resistance exercise, aerobic exercise, and flexibility exercises to improve range of motion. ${ }^{27,17}$ Circuit exercise is a program that includes all three types of exercise. Circuit exercise programs generally have two or more types of motion, alternating between them, and can be designed in a variety of protocols.

A typical circuit exercise program can include six to fifteen items, and one exercise can range from 30 seconds to 1 minute, with or without resting between stations..$^{24,28}$ It is recommended that the exercise program should be performed at least twice a week and with an intensity over moderate intensity. ${ }^{28}$ Compared to resistance exercise, circulation exercise is recommended for sarcopenic obesity elderly because it has the following advantages.24 First, there is little risk of injuries and is not limited by cost and location. Second, the participants can carry out group exercise and perform exercise for a long duration continuously with interest. Third, it shows the improvement of body composition and various physical factors in a relatively short time.

Previous studies have shown that circuit exercise for 12 weeks, 3 times a week, 50 minutes a day, and $70 \%$ target heart rate (THR) for men and women obese elderly over 65 years resulted in a positive improvement effect in body composition with decreasing body weight and fat mass and increased free fat mass.$^{29}$ Whitehurst et al., ${ }^{30}$ found in the elderly men and women subjects aged over 65 years that cycling with a intensity of $70-80 \%$ for $40-50$ minutes daily for 12 weeks, three times per week resulted in a positive improvement in stamina variables such as walking ability, leg endurance, and flexibility. In a study of Jung \& Lee., ${ }^{31}$ sarcopenia elderly women over 65 years performed circuit exercise with $60 \sim 80 \%$ intensity of HRR for 12 weeks, 3 times a week, 55 minutes a day, as a result, positive effects of metabolismrelated variables were shown by the decrease of insulin resistance and atherosclerosis index. Also, Balachandran et al., ${ }^{26}$ investigated the effects of circuit exercise program on obese elderly, sarcopenic elderly, and obese older over 65 years people with muscular dystrophy were treated with 15 weeks, twice a week, 60 minutes daily, as a result, walking ability and upper and lower strength were increased, and physical fitness was improved in all intervention (Table 1).

In summary, circulatory exercise modality is similar to the result of treating only resistance exercise, and regardless of gender and age it can be seen that exercise program is effective for improvement of health factor in elderly people who have sarcopenia, obesity or sarcopepia and obesity at the same time. Especially the improvements of body composition, physical fitness and blood variables were more effective in the elderly with increased fat mass and decreased free fat mass. In this regard, exercise intervention for sarcopenic obesity elderly is recommended with moderate intensity exercise combined with aerobic and resistance types at least 12 weeks, more than 2 times a week, more than 50 minutes daily. These exercise programs can significantly improve the health status of obese older people (Table 2). Despite these positive effects, however, the reported studies are limited. Future studies should identify the effects of strength, frequency, time, and duration of various circuit exercise program in sarcopenic obesity elderly. 
Table I Clinical circuit exercise intervention studies for older adults

\begin{tabular}{|c|c|c|c|c|}
\hline Study & Subject \& age & Duration & $\begin{array}{l}\text { Main outcome } \\
\text { measure }\end{array}$ & Results \\
\hline Whitehurst et al. ${ }^{30}$ & $\begin{array}{l}\text { Older men and women } \\
(\mathrm{n}=119) \\
73.0 \pm 4.6 \mathrm{yr}\end{array}$ & $12 \mathrm{Wk}$ & Physical fitness & $\begin{array}{l}\text { Significant improvement of } \\
\text { get up and go, sit to stand, standing reach, timed } \\
\text { walk, sit and reach, physical function }\end{array}$ \\
\hline Bocalini et al. ${ }^{29}$ & $\begin{array}{l}\text { Obese women elderly } \\
(\mathrm{n}=69) \\
(\mathrm{AWC}, 67 \mathrm{yr} \text { AWT, } 66 \mathrm{yr} \text {, } \\
\text { OWC, } 63 \mathrm{yr}, \text { OWT, } 64 \\
\text { yr, OC, } 62 \mathrm{yr}, \text { OT, } 62 \mathrm{yr})\end{array}$ & $12 \mathrm{Wk}$ & Body composition & $\begin{array}{l}\text { Significant decrease of body weight, fat mass and } \\
\text { significant increase of free fat mass in overweight } \\
\text { and obesity training group }\end{array}$ \\
\hline Jung \& Lee ${ }^{31}$ & $\begin{array}{l}\text { Sarcopenia women } \\
\text { elderly }(\mathrm{n}=24) \\
\text { CE group }(74.4 \pm 1.1) \\
\text { C group }(73.5 \pm 1.4)\end{array}$ & $12 \mathrm{Wk}$ & $\begin{array}{l}\text { Daily living } \\
\text { fitness, iso kinetic } \\
\text { function, } \\
\text { biochemical } \\
\text { property of } \\
\text { muscle }\end{array}$ & $\begin{array}{l}\text { Significant improvement of grip strength, upper } \\
\text { arm flexion, sit-and-reach, one leg standing } \\
\text { with eyes-open, up and go, maximal oxygen } \\
\text { consumption, and peak torque } \\
\text { per body weight, average power at extension and } \\
\text { flexion, and IL-6 of CE group }\end{array}$ \\
\hline $\begin{array}{l}\text { Balachandran et } \\
\text { al. }{ }^{26}\end{array}$ & $\begin{array}{l}\text { Sarcopenic obese men } \\
\text { and women elderly } \\
(\mathrm{n}=17) \\
\mathrm{SH}(71.0 \pm 8.2) \\
\mathrm{HSC}(71.6 \pm 7.8)\end{array}$ & $15 \mathrm{Wk}$ & physical function & $\begin{array}{l}\text { Significant improvement of muscle power, } 1 \mathrm{RM} \\
\text { in leg and chest, sit \& stand, pan carry of HSC }\end{array}$ \\
\hline
\end{tabular}

Note AW, appropriate weight; OW, overweight; O, obese; control,AW;AWC, trained;AWT; OWC, OW control; OWT, trained:; OC, O control; OT, O trained; CE group, circuit exercsie group; $\mathrm{C}$ group, control group; HSC, high-speed circuit training group; $\mathrm{SH}$, traditional strength/hypertrophy training group.

Table 2 Circuit training program recommendations

\begin{tabular}{ll}
\hline & $\begin{array}{l}\text { Resistance exercise-weight load, elastic bands, dumbbell and weight machines, atc. } \\
\text { Aerobic exercise-cycling, treadmill, walking, running, skipping, stair climbing, atc. } \\
\text { Core exercise-plank, floor bridge, marching, atc. } \\
\text { Flexibility exercise }\end{array}$ \\
Intensity & $8-15$ RM of 1RM, 50-80\% of HRR(heart rate reserve), 12-15 of RPE(Rating of Perceived Exertion) \\
Frequency & $1 \sim 3$ days per week with at least 48 hours between sessions \\
Duration & Less than 1 hour \\
& $\begin{array}{l}\text { Number of Stations 6-15 } \\
\text { Number of circuits per session minimum of 1, preferably 2-3 } \\
\text { Method of exercise }\end{array}$ \\
& $\begin{array}{l}\text { Time of circuits training session 30-90 seconds } \\
\text { Rest Interval between Circuits 2-3 minutes }\end{array}$ \\
\end{tabular}

\section{Conclusion}

Sarcopenic obesity has a negative effect on the metabolismrelated conditions such as decreased body function and physical fitness, blood lipids, inflammation in the body and hormones in elderly. Exercise therapy is recommended as a method to solve this problem. In general, recommended exercise type for the elderly is resistance exercise type to improve muscle strength, but it does not improve body composition such as body fat mass. Therefore, circuit exercise modality combined with resistance and aerobic exercises shows improving effect on body composition, physical fitness, and metabolic parameters are recommended rather than just resistance exercise program. The circuit exercise using weight load is less risk of injuries and can be continued with interest without restriction of cost and place. Recommended exercise method for improvement of health related factors in sarcopenic obesity elderly should be at least 12 weeks, more than 2 times a week, more than 50 minutes daily, and higher than moderate intensity. However, despite these positive studies, the studies reported so far are very limited and future studies should identify the effects of strength, frequency, time, and duration of various circuit exercise program in sarcopenic obesity elderly.

\section{Acknowledgements}

This work was supported by the Ministry of Education of the Republic of Korea and the National Research Foundation of Korea (NRF-2016S1A5B5A07920341).

\section{Conflict of interest}

The author has no conflict of interest to report.

\section{References}

1. Morley JE, von Haehling S, Anker SD, et al. From sarcopenia to frailty: a road less traveled. J Cachexia Sarcopeni. 2014;5(1):5-8.

2. Bouchonville MF, Villareal DT. Sarcopenic Obesity-How Do We Treat It?. Curr Opin Endocrinol Diabetes Obes. 2013;20(5):412-419.

3. Liu CJ, Latham NK. Progressive resistance strength training for improving physical function in order adults. Cochrane Database Syst Rev. 2009;3:CD002759.

4. Ministry of Health \& Welfare. Korea National Health and Nutrition Examination Survey (KNHANES). 2017. 
5. McGlone ER, Bond A, Reddy M, et al. Super-Obesity in the Elderly: Is Bariatric Surgery Justified?. Obes Surg. 2015;25(9):1750-1755.

6. Luppino FS, Wit LM, Bouvy PF, et al. Overweight, obesity, and depression: a systematic review and meta-analysis of longitudinal studies. Arch Gen Psychiatry 2010;67(3):220-229.

7. Taylor VH, Forhan M, Vigod SN, et al. The impact of obesity on quality of life. Best Pract Res Clin Endocrinol Metab. 2013;27(2):139-146.

8. Esser N, Legrand PS, Piette J, et al. Inflammation as a link between obesity, metabolic syndrome and type 2 diabetes. Diabetes Res Clin Pract. 2014;105(2):141-150.

9. Williams RS, Heilbronn LK, Chen DL, et al. Dietary acid load, metabolic acidosis and insulin resistance-Lessons from cross-sectional and overfeeding studies in humans. Clin Nutr. 2016;35(5):1084-1090.

10. Singhal M, Vishnu VM, Raju SR, et al. Interrelationship between obesity and cancer. Kidney (renal-cell). 2013;1(2.5):42-45.

11. Rosenberg IH. Sarcopenia: origins and clinical relevance. $J$ Nutr. 1997;127(5):990S-991S.

12. Siparsky PN, Kirkendall DT, Garrett WE. Muscle changes in aging: understanding sarcopenia. Sports Health. 2014;6(1):36-40.

13. Rom O, Kaisari S, Aizenbud D, et al. Lifestyle and sarcopeniaetiology, prevention, and treatment. Rambam Maimonides Med J. 2012;3(4):1-12.

14. Goodpaster BH, Park SW, Harris TB, et al. The loss of skeletal muscle strength, mass, and quality in older adults: the health, aging and body composition study. J Gerontol A Biol Sci Med Sci. 2006;61(10):10591064.

15. Baumgartner RN, Koehler KM, Gallagher D, et al. Epidemiology of sarcopenia among the elderly in New Mexico. Epidemiol Rev. 1998;147(8):755-763.

16. Cruz JAJ, Baeyens JP, Bauer JM, et al. Sarcopenia: European consensus on definition and diagnosisReport of the European Working Group on Sarcopenia in Older People. Age and ageing. 2010, 39(4): 412-423.

17. Ryan AS. Exercise in aging: its important role in mortality, obesity and insulin resistance. Aging Health. 2010;6(5):551-563.

18. Brooks N, Layne JE, Gordon PL, et al. Strength training improves muscle quality and insulin sensitivity in Hispanic older adults with type 2 diabetes. Int J Med Sci. 2007;4(1):19-27.
19. Strasser B, Schobersberger W. Evidence for resistance training as a treatment therapy in obesity. J Obes. 2011;2011:1-9.

20. Lima RM, Bezerra L, Rabelo HT, et al. Fat-free mass, strength, and sarcopenia are related to bone mineral density in older women. J Clin Densitom. 2009;12(1):35-41.

21. Verdijk LB, Gleeson BG, Jonkers RA, et al. Skeletal muscle hypertrophy following resistance training is accompanied by a fiber type-specific increase in satellite cell content in elderly men. J Gerontol A Biol Sci Med Sci. 2009;50(1):1-8.

22. Kryger Al, Andersen JL. Resistance training in the oldest old: consequences for muscle strength, fiber types, fiber size, and MHC isoforms. Scand J Med Sci Sports. 2007;17(4):422-430.

23. Kraemer WJ, Ratamess NA. Fundamentals of resistance training: progression and exercise prescription. Med Sci Sports Exerc. 2004;36(4):674-688.

24. Rasmussen CR, Kreider C, Kerksick B, et al. Effects of the curves? fitness and weight loss program on markers of health. Med Sci Sports Exerc. 2004;36(5):106-112.

25. Contrò V, Bianco A, Cooper J, et al. Effects of different circuit training protocols on body mass, fat mass and blood parameters in overweight adults. J Biol Res. 2017;90(1):6279.

26. Balachandran A, Krawczyk SN, Potiaumpai M, et al. High-speed circuit training vs hypertrophy training to improve physical function in sarcopenic obese adults: a randomized controlled trial. Exp Gerontol. 2014;60:64-71.

27. ACSM. ACSM'S Guidelines for Exercise Testing and Prescription. 9th ed. Lippincott, Williams \& Wilkins: Philadelphia, PA, 2014.

28. Fleck SJ, Kraemer WJ. Designing Resistance Training Programs. 3rd ed. Champaign, IL: Human Kinetics; 2004.

29. Bocalini DS, Lima LS, Andrade SD, et al. Effects of circuit-based exercise programs on the body composition of elderly obese women. Clin Interv Aging. 2012;7:551-556.

30. Whitehurst MA, Johnson BL, Parker CM, et al. The benefits of a functional exercise circuit for older adults. J Strength Cond Res. 2005;19(3):647-651.

31. Jung WS, Lee MG. Effects of 12 weeks of circuit training on body composition and surrogate indices of major lifestyle disease in sarcopenia elderly women. Korean J Sports Sci. 2017;26(6):11131124. 